\title{
Colloidal Quantum Dots enabling Coherent Light Sources for Integrated Silicon-Nitride Photonics
}

\author{
Weiqiang Xie, Yunpeng Zhu, Suzanne Bisschop, Tangi Aubert, Zeger Hens, Dries van Thourhout, \\ member, IEEE, Pieter Geiregat, member, IEEE
}

\begin{abstract}
Integrated photonic circuits, increasingly based on silicon (-nitride), are at the core of the next generation of low-cost, energy efficient optical devices ranging from on-chip interconnects to biosensors. One of the main bottlenecks in developing such components is that of implementing sufficient functionalities on the often passive backbone, such as light emission and amplification. A possible route is that of hybridization where a new material is combined with the existing framework to provide a desired functionality. Here, we present a detailed design flow for the hybridization of silicon nitride - based integrated photonic circuits with so-called colloidal quantum dots (QDs). QDs are nanometer sized pieces of semiconductor crystals obtained in a colloidal dispersion which are able to absorb, emit and amplify light in a wide spectral region. Moreover, they combine costeffective solution based deposition methods, ambient stability and low fabrication cost. Starting from the linear and non-linear material properties obtained on the starting colloidal dispersions, we can predict and evaluate thin film and - device performance, which we demonstrate through characterization of the first onchip QD based laser.
\end{abstract}

Index Terms - Integrated Optics, Nanotechnology, Quantum Dots, Silicon on insulator technology

\section{INTRODUCTION}

Silicon photonics holds great promise for future integrated optical networks where photons instead of electrons are used to carry information, either across the globe or on a microchip.[1] Using silicon, it can rely on the immense CMOS fabrication expertise and infrastructure. Recent years saw the commercialization of several silicon photonics products from major industrial players such as IBM, Alcatel-Lucent or Intel. The possibility to fabricate small footprint modules with low energy consumption on a large scale is not only interesting for the long-range telecom market. Applications in fields as diverse as lab-on-a-chip biosensing, quantum computing and on-chip interconnects are intensely explored.[2] Although the use of silicon based (nano-)photonics brings unique advantages in terms of scalability and end-cost, it does put a limit to the functionalities that can be achieved. Indeed, although silicon is an excellent light guide due to its high refractive index, it falls short as a material for generating light due to its indirect band gap. This makes that crucial integrated photonic building blocks such as an efficient coherent light

\footnotetext{
${ }^{\mathrm{M}}$ anuscript received November 29, 2016. P. Geiregat is with the Department of Inorganic and Physical Chemistry and the Department of Information Technology, Ghent University, Belgium, email: pieter.geiregat@ugent.be ; Tel: +329264 4864 W. Xie, Y.Zhu and S. Bisschop are with the Department of
}

source (i.e., a laser) are notoriously difficult to make from plain silicon.[3]-[5]In addition, silicon's relatively narrow band gap limits the use of the platform to long wavelengths and strong two-photon absorption pushes this range even further for high power non-linear applications. An alternative is to use silicon nitride ( $\mathrm{SiNx})$, a dielectric with a large band gap (ca. $5 \mathrm{eV}$ ) that can be processed within the same CMOS framework as silicon. Light emission and absorption becomes even more difficult due to the dielectric nature of $\mathrm{SiNx}$, yet the large gap opens up possibilities of integrated photonics in the visible part of the spectrum where on-chip bio-sensing and Raman spectroscopy are actively investigated.[6] The most common approaches to remedy the shortcomings of silicon (nitride) for light emission and amplification are (a) bonding or (b) direct growth of functional materials/circuits on top of silicon. [7], [8] Recently, the direct growth procedure resulted in the first electrically pumped continuous wave laser directly grown on silicon using epitaxially grown QDs.[9] An alternative approach is that of hybridization with solution processable materials, where a desired material is deposited on silicon using low temperature and non-vacuum deposition methods such as spin coating. A possible, yet for now rather unexplored, way of achieving this is the use of inorganic colloidal quantum dots (QDs)[10]-[12], i.e., semiconductor nanocrystals obtained through a wet chemical synthesis. The optical properties, such as light absorption and emission, are optimized by exploiting various parameters such as particle composition and architecture (core/shell, shape/size, hybrid metallic/semiconducting). A broad absorption spectrum combined with a narrow photoluminescence, tunable from the UV to the mid-infrared with a high photoluminescence quantum yield makes them very appealing for applications in opto-electronics. Additionally, the low cost of the QD synthesis and deposition methods, typically through nonvacuum techniques such as spin coating or Langmuir-Blodgett deposition, already puts them at the forefront of diverse applications such as displays, lighting, solar cells and luminescent solar concentrators.[13] The same assets of tunable optical properties and easy processing makes QDs most appealing for hybrid integrated photonics, where they could be used as building blocks for coherent light sources such as lasers or incoherent broadband LEDs. Several groups have worked combining QDs with integrated photonic

Information Technology, Ghent University, Belgium. Z.Hens, S. Bisschop and T. Aubert are also with the Department of Inorganic and Physical Chemistry, Ghent University. 
circuits, mainly focusing on combining QDs with microcavities or plasmonic components to boost the spontaneous emission rate through a Purcell-effect. [14]-[19] In this review, we will focus on the development of coherent integrated light sources based on colloidal QDs and expand on a design methodology for such components. In particular, we will focus on the interplay betwen the requirements of the integrated component and the implications for the QD's linear and non-linear properties. Two prime demonstrators will be used to demonstrate the validity of our approach: waveguidebased amplifiers and microdisk lasers for the silicon-nitride platform fabricated with flash CdSe/CdS QDs. [20] Even though we focus on the state-of-the art flash QDs, the design flow presented here is generic and can in principle be applied to future solution-processable colloidal materials such as the promising 2D 'nanoplatelets' or Cd-free quantum dots such as $\mathrm{InP} / \mathrm{ZnS}(\mathrm{e}) .[21]-[23]$

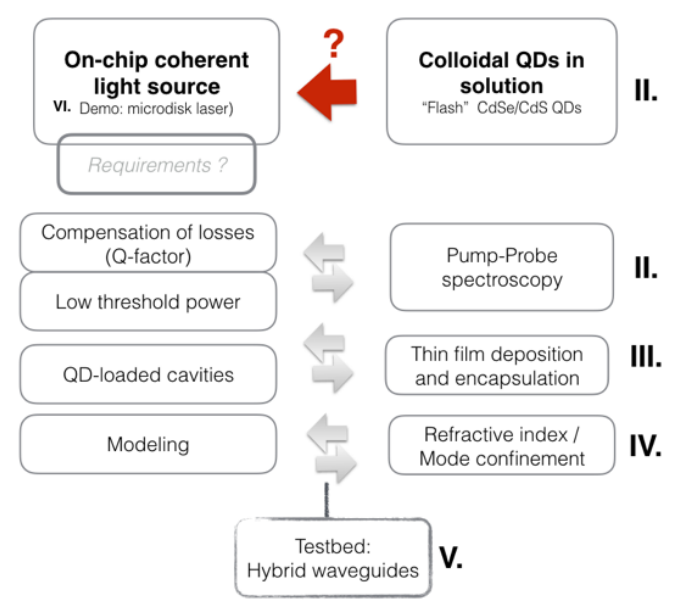

Fig. 1. Design methodology for QD-enabled on-chip devices

This review presents the whole flow of translating the exciting solution-based properties of colloidal QDs (efficient spontaneous emission, broadband optical gain, solution processability, ..) into the community of integrated photonic components. A toolbox is presented that allows to connect and combine the output of one community (optical properties of colloidal QDs) to the requirement of the other (integrated device requirements).

The flow (see also Figure 2) is based on a number of distinct steps:

- Section II: Obtaining the relevant linear and nonlinear material properties of colloidal QDs in solution, judging their quality and developing a framework to translate the properties into devicespecific quantities.

- Section III: Transfer of QDs from the colloidal solutions to (patterned) thin films with high uniformity, followed by embedding of the QDs into SiNx stacks and etching said stacks into desired onchip components, without degradation of material quality and non-linear properties (especially light amplification).

- Section IV: Develop a theory framework to couple the solution based properties to 'modal' properties of the integrated components.

- Section V: Hybrid waveguides will be developed as a testbed to characterize the latter.

- Section VI: Using the above to design and fabricate a photonic component, in particular an integrated microdisk laser, and couple the output back to possible optimization of the previous steps, e.g. QDs with higher modal gain, optically smoother films, stronger mode coupling, ...

\section{Colloidal Quantum Dots}

Opposite from epitaxially grown semiconductors, colloidal QDs are obtained as a dispersion of nanocrystals in an apolar solvent through wet chemical synthesis (see Fig. 2).[12] Their linear optical properties, such as absorption and spontaneous emission, are therefore usually evaluated on such dispersions. We will extend this idea and show that nearly every important material property can be evaluated from such solution-based measurements: the linear but also non-linear absorption / emission spectra, material gain, optical gain threshold and the excited state lifetime for stimulated emission.

\section{A. CdSe/CdS core/shell quantum dots}

The most-studied colloidal QD systems are most likely the Cd-based core and CdSe/CdS core/shell QDs, as these materials are active in the visible part of the spectrum and can be synthesized with extreme control over their size and shape.[20] Coating CdSe cores with a wide gap material allows to tune the opto-electronic properties and also provides a better passivation for the core resulting in high photoluminescence quantum yields. In our approach, $\mathrm{CdSe} / \mathrm{CdS}$ QDs are synthesized through a seeded growth flash procedure [20], [24] which rapidly yields core/shell QDs with a sizeable shell. Fig. 2 shows a Transmission Electron Microscope (TEM) image of such flash QDs and their respective linear absorption and emission spectrum when dispersed in toluene, respectively. High photoluminescence quantum yields (routinely $50-70 \%$, with record values of $80 \%$ ) and narrow linewidths (FWHM of $30 \mathrm{~nm}$ ) are obtained, even for very thick CdS shells. Typical radiative lifetimes of $c a .60$ ns are obtained after non-resonant photo-excitation.

Suppressed non-radiative Auger recombination and reduced single QD blinking were observed, possibly due to the formation of an alloy at the CdSe/CdS core/shell interface leading to a smoother confinement potential. [25]-[27] As we will see further, these QDs can be deposited on silicon(nitride) with monolayer precision and, importantly, they retain their optical properties even after embedding into SiNx, in part due to the thick CdS shell which protects the emitting core from degradation (see section III). Further improvements could be made by coating the CdSe/CdS QDs with a ZnS(e) shell [20] which further protects the core exciton states from surface mediated non-radiative relaxation.[28] 

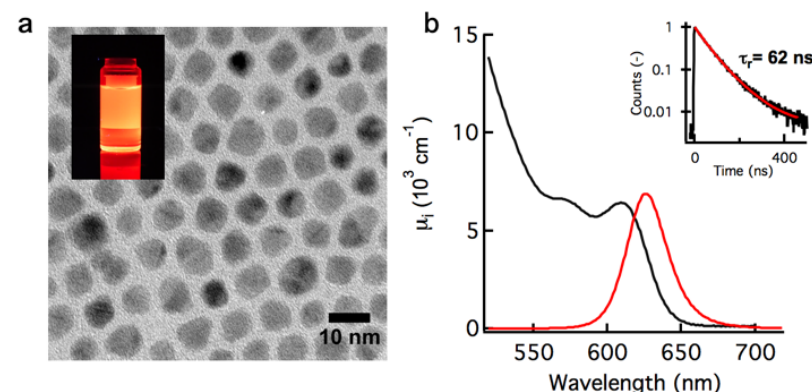

Fig. 2. Overview of flash CdSe/CdS QDs (a) TEM image of CdSe/CdS core $/$ shell QDs with a core size of $3.5 \mathrm{~nm}$ and a total size of $8 \mathrm{~nm}$. Inset: $\mathrm{CdSe} / \mathrm{CdS}$ QDs in toluene under UV illumination (b) Absorption (black, expressed as intrinsic absorption coefficient $\mu_{i}$, see part II.B) and emission (red) spectrum after excitation with $365 \mathrm{~nm}$ with a luminescence quantum yield of $80.8+/-3.6 \%$. Inset: Photoluminescence (PL) decay at $620 \mathrm{~nm}$ showing an average decay time of $62 \mathrm{~ns}$.

\section{B. Intrinsic Material Properties}

The most obvious linear property of QDs is that of broadband light absorption. We will present a framework to use this ubiquitous property to predict more intricate properties (see section II.C) such as the material gain. Characterizing a colloidal dispersion of QDs by the QD volume fraction $f$, we can write the extinction coefficient of that composite medium as the product of an intrinsic absorption coefficient $\mu_{i}\left(\mathrm{~cm}^{-1}\right)$ and the volume fraction $f[29]$ :

$$
\mu=\mu_{i} \times f
$$

In this framework, $\mu_{i}$ is the absorption coefficient of a fictitious QD dispersion that has a QD volume fraction of 1 . In the case of simple core-only systems, one can write $\mu_{i}$ using Maxwell-Garnett theory as:

$$
\mu_{i}(\lambda)=\frac{2 \pi}{\lambda}\left|f_{L F}\right|^{2} \operatorname{Im}\left(\varepsilon_{Q D}\right)
$$

Here, $\lambda$ is the wavelength of interest, $\varepsilon_{Q D}$ is the complex dielectric function of the QD material and $\left|f_{L F}\right|$ is the local field factor which takes screening of the external field due to the dielectric mismatch between QD core and the environment into account (see also section IV). For many quasi-spherical QDs, it has been found that $\mu_{i}$ is size-independent at short wavelengths, with an experimentally verified value closely matching the value predicted by eq. 2 when using the bulk dielectric function of the QD constituent material. [5] For materials with different shapes or core/shell composition, eq. 2 must be replaced by more elaborate expressions, yet it has been found that the same short wavelength limit applies.[30], [31] In the case of core/shell QDs such as CdSe/CdS [32], this makes that $\mu_{i}$ becomes a function of the geometry of the particle through the ratio of shell volume to total volume (see Fig. 3). [33] When the dielectric environment of the QDs changes, e.g. upon film formation, the local field factor in eq. 2 might change slightly. Typically, an increase in the permittivity of the QD environment leads to an increase of the local field factor and hence a slightly larger $\mu_{i}$ could be expected, e.g. in thin films.
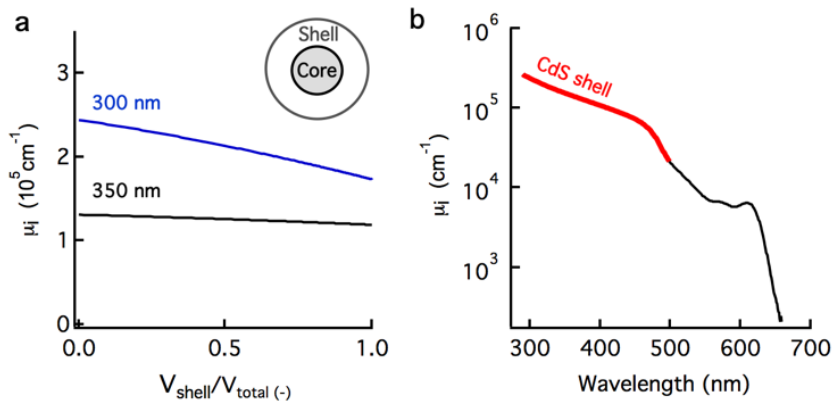

Fig. 3. (a) Calculated intrinsic absorption coefficient of wurtzite $\mathrm{CdSe} / \mathrm{CdS}$ in toluene at 300 and $350 \mathrm{~nm}$ for varying composition and (b) $\mu_{i}$ spectrum of a typical CdSe/CdS QD on a logarithmic scale showing the contribution of the strong CdS shell absorption at shorter wavelengths. Dielectric constants were taken from Adachi [34], which e.g. at $300 \mathrm{~nm}$ amounts to $\varepsilon_{C d S e}=7.6+6.7 i$

$$
; \varepsilon_{C d S}=6.6+3.4 i \text { ). The solvent (toluene) was taken as } \varepsilon_{t o l}=2.2 \text {. }
$$

The approach outlined above allows us to calculate a $\mu_{i}$ spectrum from a regular absorbance spectrum $A(\lambda)$, regardless of the QD volume fraction (see Fig. 3b) once the value for $\mu_{i}$ is known at a reference wavelength $\lambda_{r}$ (e.g. at 300/350 nm for CdSe/CdS, see Fig. 3):

$$
\mu_{i}(\lambda)=A(\lambda) / A\left(\lambda_{r}\right) \times \mu_{i}\left(\lambda_{r}\right)
$$

It is important to realize that $\mu_{i}$ is a quantity depending on both the optical properties of the material at hand and the local environment. Neglecting the latter dependence in a first approximation yields a reasonable estimate of the optical properties of a QD film (characterized by a QD volume fraction $f$ ) or a specific photonic device (characterized by a modal overlap $\Gamma$ ). When needed, the influence of local field effects in thin films (see Eq. 2) can be accounted for in more detailed calculations, see section IV.[35]

\section{Optical Properties under Strong Photoexcitation}

Although the linear optical properties discussed in the previous sections already prime QDs for applications as diverse as on-chip (spontaneous) light emission and absorption, the step to high end applications such as amplifiers and lasers requires knowledge of the optical response under strong photo-excitation. Indeed, we aim at determining the conditions needed to develop stimulated emission and hence net optical gain. Again, we will show that we can characterize this 'non-linear' response through measurements on colloidal QD dispersions as obtained directly from the chemical synthesis. Several types of colloidal QDs have shown net optical gain since the first demonstration over 15 years ago for core-only CdSe QDs.[36] A bottleneck in the development of colloidal QDs for optical gain is the tradeoff between their size and non-radiative recombination processes such as surface trapping and Auger recombination which quench the excited state population required for optical gain.[37], [38] Indeed, due to the finite degeneracy of the discrete band edges in QDs, stimulated emission and hence optical gain arise from bi-exciton-to-exciton transitions. Unfortunately, the bi-exciton state is subject to the mentioned Auger process, leaving it to vanish on timescales of a few $10-100$ ps in core-only 
QDs.[39] Using CdSe/CdS, it was demonstrated that both a smooth interface potential between core and shell and an increased exciton volume could lead to a strong increase of the Auger lifetime, even to the level of several nanoseconds. [27], [40] Type II QDs which rely on strong carrier-carrier interactions to achieve a lower gain threshold [41] are not very practical because one of the charges (e.g. the hole in the reference system $\mathrm{CdS} / \mathrm{ZnSe}$ ) is pushed to the surface where it can be trapped quite easily by surface defects.[42]

One of the difficulties in designing devices relying on optical gain (amplifiers \& lasers) is that the conditions under which the QD material itself can develop sufficient stimulated emission (i.e. to counter the inevitable optical losses in integrated devices), are not well known. Such assessments involve an estimate of the modal gain, which would need the QD material gain as an input. Here, we will show that this desired material gain, labeled $g_{i}$ in this work, can be measured using transient absorption spectroscopy and we will demonstrate (see section IV.-V.) that it provides a good estimate of the experimental modal gain of $\mathrm{SiNx} / \mathrm{QD} / \mathrm{SiNx}$ waveguides.

Transient absorption spectroscopy (TAS) is a pump-probe technique where a sample, e.g. a dispersion of colloidal QDs, is pumped with ultrashort laser pulses after which the change in absorption $\Delta A$ is measured by a time-delayed probe pulse as function of wavelength (or energy). Fig. 4a shows an example of a $2 \mathrm{D} \triangle A$ - map obtained in a broadband TAS experiment. Here, $\Delta A$ is shown both as function of the probe wavelength (horizontal axis) and time delay (vertical axis). In particular, a $\mathrm{CdSe} / \mathrm{CdS}$ QD dispersion is pumped using $110 \mathrm{fs}$ pump pulses at $520 \mathrm{~nm}$ with a $1 \mathrm{kHz}$ repetition rate. The most striking feature is a reduction in the absorption or bleach $(\Delta A<0)$ at around 590 and $620 \mathrm{~nm}$ due to state filling of the band-gap transitions of the CdSe core.[43]

Combining $\triangle A$ with the linear absorption spectrum $A_{0}$ of unexcited QDs, shown in black in Fig. 4a, yields at every delay time the actual absorbance $A$ of the dispersion after photo-excitation. The concomitant map of $A$ is shown in Fig. $4 \mathrm{~b}$ for negative values of $A$ only. Indeed, when $A$ turns negative, probe light is actually amplified. Note that this is not amplified spontaneous emission, it simply implies that the probe beam is 'amplified' instead of absorbed. We clearly observe such a state of 'population inversion' or net stimulated emission in the region from 560 to $660 \mathrm{~nm}$ lasting for the first ca. $10-100$ ps after the pump pulse. Fig. $4 \mathrm{c}$ represents nonlinear absorbance traces obtained on the same sample at a fixed probe wavelength of $620 \mathrm{~nm}$ at different pump pulse energies. It can be seen that a minimum pulse energy is needed to reach $A<0$ or population inversion, a quantity we call the gain threshold $P_{t h}$. However, once pump energies are sufficiently high, population inversion can be reached within 1 ps after pumping and can last up to $c a .650 \mathrm{ps,} \mathrm{a} \mathrm{time} \mathrm{we} \mathrm{call}$ the 'inverted state lifetime' or 'gain lifetime' $\tau_{g}$. Using eq. 3 , the absorbance $A$ at a given time delay is quickly rescaled into the intrinsic gain or material gain $g_{i}$ (see Figure $4 \mathrm{~d}$ ). This number yields the gain a QD material with a volume fraction $f$ $=1$ would have under the same pump conditions. Multiplying it with the actual volume fraction in a given situation yields the gain coefficient of any specific QD sample, from a closepacked thin film to dilute polymer mixtures. Fig. $4 \mathrm{~d}$ shows the material gain $g_{i}$ - spectra at a 5 ps time delay. Especially at higher pulse energies, a broad gain band band develops with $g_{i}$ reaching values of up to $2000 \mathrm{~cm}^{-1}$. As typical loss values for $\mathrm{SiNx}$-based micro-resonators and waveguides are in the range of $1-100 \mathrm{~cm}^{-1}$, these $g_{i}$ numbers clearly indicate the potential for QDs to enhance SiNx photonics. [44] Finally, the inset of Fig. 4d shows the material gain for increasing fluence allowing us to define a gain threshold which can be expressed in energy $/ \mathrm{cm}^{2}$ (here $140 \mu \mathrm{J} / \mathrm{cm}^{2}$ ) or by means of the number of absorbed photons per QD, here labeled $\langle N\rangle$. As expected from the bi-exciton nature of optical gain in colloidal QDs, the threshold value $\langle N\rangle_{t h} \cong 1$.[41]
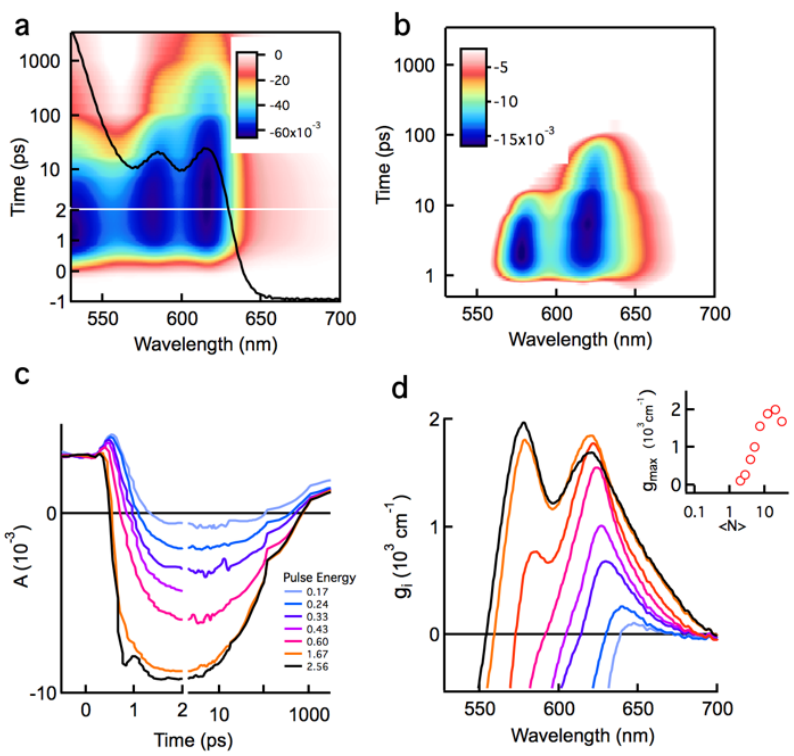

Fig. 4. Overview of multi-excitonic properties in solution measured using pump-probe spectroscopy for a dispersion of $\mathrm{CdSe} / \mathrm{CdS}$ with a core size of 4 $\mathrm{nm}$ and a total size of $9 \mathrm{~nm}$ (a) 2D time-wavelength map of $\Delta A$ after $520 \mathrm{~nm}$ photo-excitation for typical CdSe/CdS QDs dispersed in toluene (b) 2D map of the absorbance $A=\Delta A+A_{0}$, where only $A<0$ is shown, i.e. the region where net stimulated emission or optical gain occurs. (c) Kinetics of the nonlinear absorption for increasing pump fluence. The net gain $(A<0)$ persists for $600 \mathrm{ps}$. (d) Material gain $g_{i}$ spectra at $5 \mathrm{ps}$ for different pump fluences (similar to $4 \mathrm{c}$ ) reaching up to $2000 \mathrm{~cm}^{-1}$. Inset shows the evolution of the maximum material gain with pump fluence, expressed as number of absorbed photons per QD,$<N>$.

To conclude, we have shown that a TA - measurement on a QD dispersion can determine: the material gain, the excited state lifetime and the gain threshold. It is important to realize that these are optimal numbers: incorporating the QDs into a cavity or waveguide will only reduce the gain coefficient (due to dilution $(f<1)$ and a finite modal confinement $(\Gamma<$ $100 \%$ )[45] and raise gain thresholds (due to guiding losses). Indeed, we can readily translate the material gain into a modal gain $g_{m}$ (see further, section IV) using device specific values for $f$ and $\Gamma$. Determination of the material gain, gain lifetime and threshold from a solution measurement thus provides us with input for a lower limit to the acceptable loss and confinement for developing nanophotonic components. 


\section{ThIN FILMS: PATTERNING, EMBEDDING \& ETCHING}

As was shown in section II, solution based measurements allow us to characterize the linear (absorption/emission, quantum yield (QY), radiative lifetime) and 'non-linear' (material gain, threshold, excited state lifetime, ...) properties of QDs. Yet, functionalizing SiNx photonics using QDs also requires a process technology, involving QD deposition, their embedding into a SiNx matrix and method for patterning those structures into (nano-)photonic components. A priori, it is not clear whether the exciting properties of high emission QY and broadband optical gain can be transferred to an embedded and patterned QD-SiNx composite. We will show that we (a) can fabricate such hybrid composites with high quality and (b) have developed a testbed to characterize them afterwards. In doing so, we can be assured that the linear and multi-excitonic properties are indeed well preserved before we make the transition to actual integrated devices.

\section{A. Film formation}

Transferring colloidal QDs from a dispersion to a thin film is, in principle, relatively quick and cost-effective as we do not need vacuum techniques. Indeed, we can use solution-based techniques such as spin coating. However, using spin coating to define optically smooth layers with monolayer control over thickness, is not so easy and hence for very thin layers, we prefer to use Langmuir-Blodgett deposition.[46] During this process (see Fig. 5a) QDs, which are hydrophobic due to the organic apolar ligand shell, are initially spread on a water surface where they quickly form a sub-monolayer. Upon compression of this sub-phase, a close packed layer can form which is then transferred to a suitable substrate using a pullout procedure (LB) and/or stamping (Langmuir-Schaeffer). [46] For either very thick or extremely dilute active layers (e.g. for single QD deposition), spin coating is often more efficient. For example, the laser cavities formed in section VI require thick active layers of over $50 \mathrm{~nm}$ and in such a case, spin coating is preferred. However, sequential use of the LB technique (or using a variation of 'stamping', so-called Langmuir-Schaefer) would allow to engineer the thickness of the QD layer with monolayer control to further meticulously optimize the performance of such devices. [47] An example of this approach is shown in Figure 8 for $\mathrm{PbS} / \mathrm{CdS}$ QDs.

\section{A. Embedding into silicon nitride}

QDs can be deposited and patterned directly on top of optical components[48], [49], yet incorporating the QDs into the bulk of the optical component by embedding dramatically improves the modal overlap and the coupling efficiency (see section V). A typical embedding process (see Fig. 6a) involves the creation of a SiNx/QD/SiNx sandwich where care is taken during deposition to ensure (a) maximum optical quality of the nitride layers and (b) minimal material damage to the QDs. To this end, the bottom layer is typically always deposited at 270 ${ }^{\circ} \mathrm{C}$ (hereafter called H-SiNx) through a Plasma-Enhanced Chemical Vapor Deposition (PECVD) process, whereas the top layer is deposited at a relatively low temperature of $120^{\circ} \mathrm{C}$ (L-SiNx) to avoid damage to the QD layer underneat. Yet, when high quality nitride is needed, the higher temperature $\mathrm{H}-$
SiNx process could used, but one should take into account that this process does seem to affect the luminescence quantum yield of the QDs (see Fig. 6b). Additional shelling with $\mathrm{ZnS}$ could remedy this issue.[20] Another crucial element in deposition of the top nitride layer is to ensure good contact between the QD layer, which is a 'soft material', and the nitride capping. To ensure this, a mixed frequency PECVD process is developed that minimizes any stress in the nitride layer which could otherwise lead to cracking.
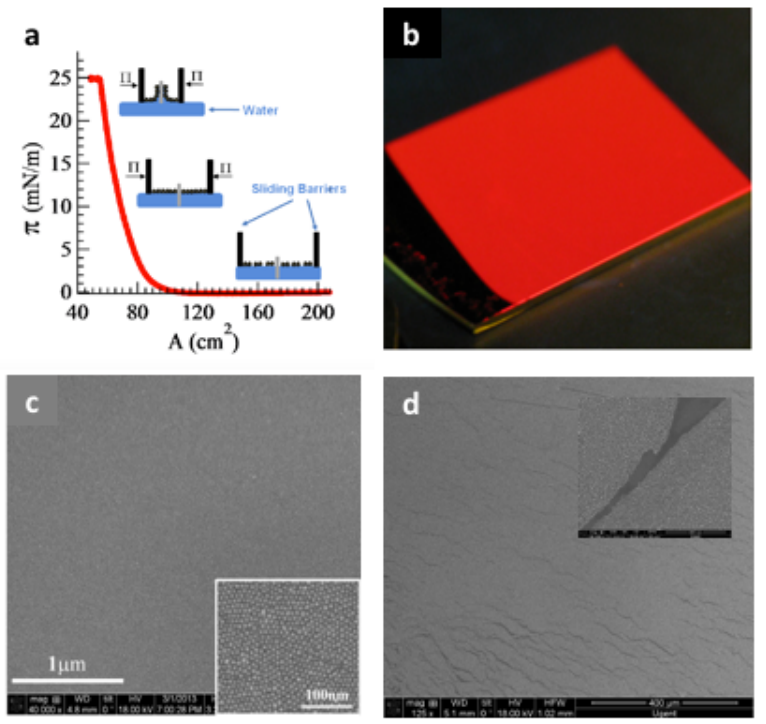

Fig. 5. Film formation (a) Schematic of Langmuir-Blodgett (LB) process (b) Quasi-perfect monolayer formed by LB deposition of flash CdSe/CdS QDs on silicon under UV illumination showing large area uniformity. (c) SEM image of the same film. (d) Possible bottlenecks in the LB process arise because of layer stress due to over-compression and (inset) cracks due to ill-passivated QDs and/or unbound ligands in the QD dispersion used for LB. Adapted in part from ref [44]. Copyright 2015 OSA
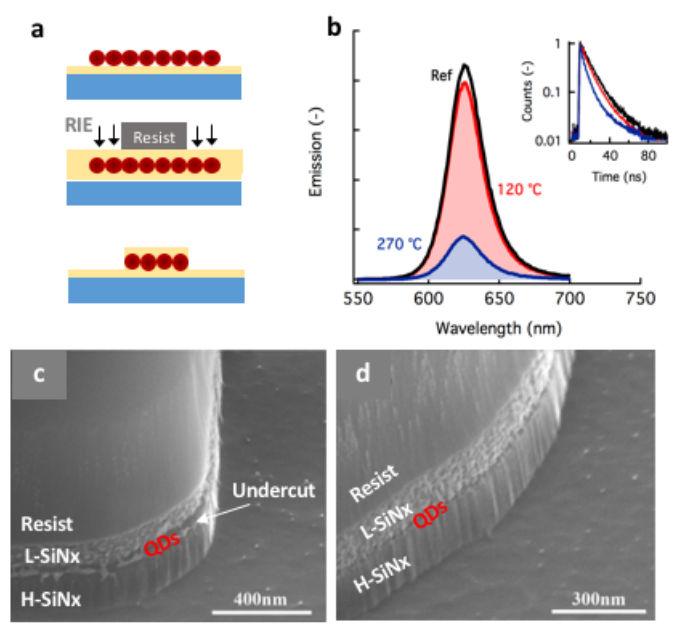

Fig. 6. Embedding and etching processes (a) Schematic of embedding and etching process where SiNx (yellow) is deposited on a QD layer (red), followed by resist patterning (grey), reactive ion etching (RIE) and resist liftoff. (b) The photoluminescence spectrum and lifetime of a ca. $50 \mathrm{~nm}$ spin coated layer of flash $\mathrm{CdSe} / \mathrm{CdS}$ QDs before and after deposition of SiNx at $270^{\circ} \mathrm{C}$ (blue) and $120^{\circ} \mathrm{C}$ (red). Clearly, the $120^{\circ} \mathrm{C}$ is more gentle for the QD layer although in both cases no spectral shifts or trap-related emission is observed. The optical quality of silicon nitride deposited at low temperature is however reduced [44], yet the effect is to be weighed with the improved preservation of the QD luminescent properties. (c) Cross section SEM of a L- 
SiNx/QD monolayer/L-SiNx stack showing the presence of an 'undercut' which is then resolved (d) by optimization of the RIE gas mixture. Adapted from ref. [44]. Copyright 2015 OSA

\section{B. Etching}

When QDs are deposited on a large area and consequently embedded into SiNx, we still need to pattern the initially 2D planar structure, e.g. into a waveguide or a microdisk (see further). We also developed patterning techniques on the level of the QDs themselves (see VI)[50], yet it is more straightforward to etch 'top down' when no specific localization of QDs within an optical component is required. Apart from material loss induced by the absorption and scattering in the nitride and/or QD film, modal losses of optical components are often limited by sidewall roughness after the etching step. Typically, a reactive ion etch (RIE) or 'dry' etch is used to obtain straight and smooth sidewalls. We optimized a gas mixture of $\mathrm{CF}_{4} / \mathrm{H}_{2}$ to etch stacks of $\mathrm{H} / \mathrm{L}$ $\mathrm{SiNx}$ [44] (see Fig. 6c,d) where the role of $\mathrm{H}_{2}$ is to form a polymer inhibitor on lateral sidewalls which protects these from further damage. The $\mathrm{H}_{2}$ - content turns out to be a key parameter to obtain smooth and straight sidewalls, in turn essential to obtain high Q microcavities (see VI). Remarkably, the RIE process does not seem to be strongly affected by the presence of an organic/inorganic QD film in between the $\mathrm{H}$ and L-SiNx, as we are able to etch through the hybrid nitride/QD stack with the same process developed for pure SiNx stacks (see Fig. 6c,d). However, a small undercut is observed in the regular process which can be alleviated by increasing the $\mathrm{H}_{2}$-content in the etching gas mixture.[51]

\section{MODELING AND THEORY}

The connection between the exciting properties measured in solution such as the material gain and the requirements of integrated nanophotonic devices such as the Q-factor is a priori not clear. This section aims to provide a starting platform to translate the intrinsic properties of QDs to device specific quantities such as the modal gain/loss by means of the 'mode confinement factor'. The latter can however only be evaluated correctly when we can model the mode profile of the hybrid QD-SiNx components.

\section{A. Refractive index of $Q D$ composites}

Simulating nanophotonic components hybridized with QDs, such as waveguides or micro-resonators, requires a means of modeling the QD layer. Typically, this is done through the use of an effective complex dielectric function $\varepsilon_{\text {eff }}$ (or effective refractive index $n_{e f f}^{2}=\varepsilon_{e f f}$ ) that models the QD layer as an effective medium [52], characterized by a volume fraction $f$ :

$$
\begin{gathered}
\varepsilon_{e f f, R} \cong \varepsilon_{h} \\
\varepsilon_{e f f, I} \cong\left|f_{L F}\right|^{2} \times f \times \varepsilon_{I, Q D}
\end{gathered}
$$

where $\varepsilon_{h}$ is the host permittivity and $f_{L F}$ is the local field factor defined as $3 \varepsilon_{h} /\left(2 \varepsilon_{h}+\varepsilon_{Q D}\right)$. Typical values for $\varepsilon_{h}$ are $\varepsilon_{h} \cong 4$ for $\mathrm{SiNx}$ and close to 1.5 for typical organic ligands and polar solvents. When SiNx is deposited onto QDs (see further), we don't expect the nitride to penetrate the QD film due to the physical nature of the CVD process as opposed to e.g. atomic layer deposition.[53] As such, when encapsulating QDs into SiNx, the host permittivity is closer to that of air/ligands, than that of SiNx (see also Fig. 8d). The complex dielectric function of the QD itself, $\varepsilon_{Q D}$, can be obtained through a procedure outlined by Moreels et al. [54] where Kramers - Kronig relations between absorption and refraction are used to determine $\varepsilon_{Q D}$ from the linear absorption spectrum of a QD composite. A viable alternative is to measure the refractive index directly through ellipsometry (Fig. 7a), where the difficulty is often to find a suitable model to extract the complex valued index from the raw ellipsometry output. [55]

\section{B. Photonic components}

Using the dispersive effective index described above, we can simulate the mode profiles of nanophotonic components through effective index solvers or full FDTD solvers. The fraction of light confined in the QD layer, the so-called 'modal confinement' $\Gamma$, will allow us to quantify light absorption and stimulated emission in a variety of photonic structures such as hybridized waveguides and cavities, see further. A useful definition of the mode confinement in a layer " $i$ " $\Gamma$ for a segmented 2D structure (e.g. a bus waveguide along the $\mathrm{z}-$ direction) is given by Visser et al. [45]:

$$
\Gamma=\frac{\int_{i}\left|\left\langle S_{z}\right\rangle\right| d S}{\int_{t o t}\left|\left\langle S_{z}\right\rangle\right| d S}
$$

where $\left\langle S_{z}(x, y)\right\rangle$ is the time-averaged z-component of the Poynting vector. We can write [56] (see also eq. 3 ):

$$
p_{m}(\lambda)=p_{i}(\lambda) \times f \times \Gamma
$$

where $f$ is the QD volume fraction and $p$ is the desired property to be evaluated such as modal optical loss or gain. $p_{i}$ is the intrinsic counterpart measured in solution such as the intrinsic absorption $\mu_{i}$ or material gain $g_{i}$ (see II.B and II.C). We will show in the next sections that this intuitive and simple formula matches very well with experimental results related to modal loss and gain, both in strip waveguides as in full high Q microcavities.

\section{Thin films}

Upon film formation, the dielectric environment of the QDs changes drastically which affects the interaction with light according to the local field factor concept. Usually, the dielectric constant of the environment (labeled $\varepsilon_{h}$ before) increases upon film formation leading to reduced screening and hence larger (modal) absorption or gain. When QDs are put in ordered superstructures, near-field dipole-dipole coupling can influence the dielectric properties to the extent that e.g. linear absorption cross sections can be enhanced depending on the particle geometry and spacing. [35] Apart from these dielectric effects, also interparticle charge transfer and Förster-type exciton transfer can occur in densely 
packed assemblies of QDs.[57] It is worth to mention that for the high quantum yield, thick shell CdSe/CdS QDs used in this work, all of these effects are often quite small as is evidenced for example by a lack of exciton red-shift or substantial quantum yield reduction upon film formation. Yet, core-only structures (such as CdSe platelets [21]) could suffer from these effects to a greater extent.
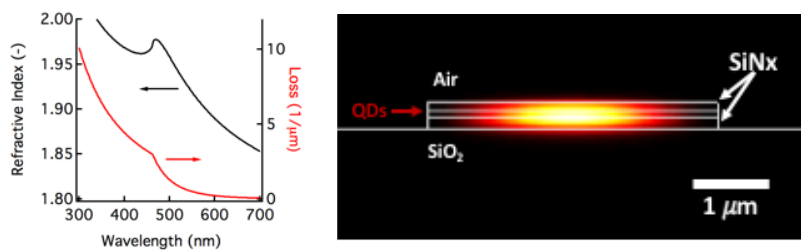

Fig. 7. (left) Refractive index and loss coefficient extracted from ellipsometry (using a Cody-Lorentz model) on a $\mathrm{SiNx} / \mathrm{QD} / \mathrm{SiNx}$ stack with a ca. $55 \mathrm{~nm}$ thick layer of flash $\mathrm{CdSe} / \mathrm{CdS}$ QDs. (right) Mode profile of fundamental TE mode in a $\mathrm{SiNx} / \mathrm{QD} / \mathrm{SiNx}$ slab structure.

\section{HYBRID WAVEGUIDES}

A useful test case for the incorporation of QDs with nanophotonics is that of simple strip waveguides. We will show that both light absorption and (stimulated) emission of QD-enabled silicon and SiNx waveguides can be achieved through the processing steps developed in section III. Moreover, we can understand the measured modal losses and net modal gain through the non-linear solution based measurements of section II and the theory framework of section IV.

\section{A. Non-embedded QDs and modal loss}

The linear optical properties of hybrid QD-functionalized have been investigated by Omari et al. [48], [58] Here, nearinfrared $\mathrm{PbS} / \mathrm{CdS}$ QDs [59] were deposited on top of siliconon-insulator ridge and rib waveguides (see Fig. 8) using Langmuir-Blodgett deposition cycles (see III.A). A first demonstration of coupling between the QDs and the optical modes of the waveguide was shown through simple transmission experiments where the waveguide loss was spectrally resolved and linked to the QD overlayer. In hybrid waveguides without embedding of the QDs, the modal confinement in the QD layer is limited to a few percent only. Fig. 8d shows that the modal loss can be traced back to the QD absorption spectrum, both qualitatively and quantitatively, indicating the validity of the classical approach of eq. 5 . Importantly, for a monolayer of QDs, the local field factor used in calculating $\mu_{i}$ uses a permittivity of the QD environment $\varepsilon_{h}$ close to 1 (air). Going to thicker layers (Fig. $8 \mathrm{~d}$, blue markers) an increased $\varepsilon_{h}$ of 1.47 is needed to find good correspondence, which can be expected from the considerations made in section II.B: reduced screening due to increase in the dielectric constant of the environment gives rise to stronger light absorption of the QD layer, i.e. a slight increase in $\mu_{i, e f f}$. We will extend the concept of eq. 4 further to also study the effect of light amplification in hybrid waveguides, where the modal gain instead of loss will be considered. The ability to model and deposit monolayer precise stacks of $\mathrm{PbS} / \mathrm{CdS}$, instead of $\mathrm{CdSe} / \mathrm{CdS}$, indicates the flexibility and generality of our approach.
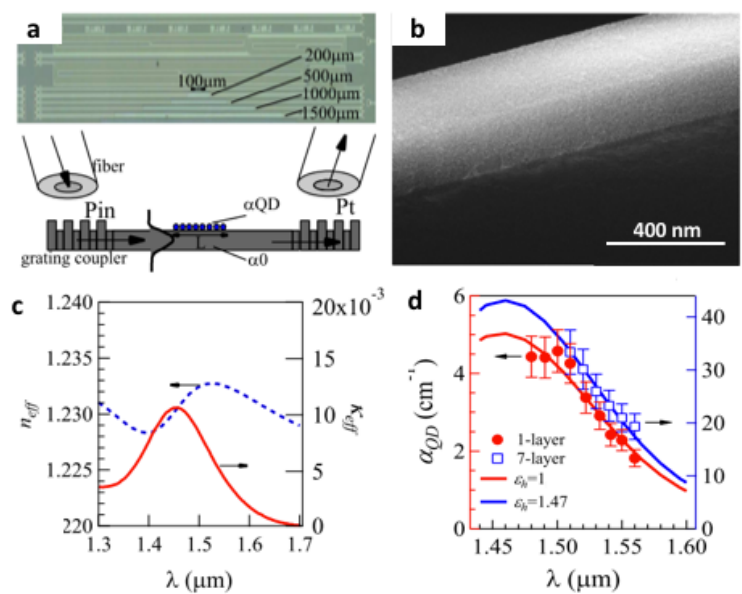

Fig. 8 Silicon-on-insulator (SOI) waveguides coated with near-infrared QDs. [60] (a) Top microscope view of SOI waveguides with optimized grating couplers. (b) SEM image of rib waveguide coated with a monolayer of $\mathrm{PbS} / \mathrm{CdS}$ QDs. (c) Effective index, real (blue dashed) and imaginary (red) part of the $\mathrm{PbS} / \mathrm{CdS}$ QD layer (d) Measured (markers) and simulated loss coefficients for different coatings of 1 and 7 monolayers. The measured loss clearly follows the absorption peak of the first exciton in $\mathrm{PbS} / \mathrm{CdS}$ QDs. Note that for the thicker 7-layer stack, a small change of the host permittivity is required, i.e. from 1 to 1.47. Adapted in part from ref. [61]. Copyright 2014 IEEE.

\section{B. Embedded QDs for Modal Gain}

We already know that the linear QD properties are well maintained upon embedding into SiNx (see III.B and Fig. 6) yet it remains unclear what effect the embedding has on the non-linear properties such as gain magnitude and threshold. Before going through the fabrication effort of a laser cavity, it is necessary to check whether also these desired multiexcitonic QD properties, i.e. the optical gain, are still preserved when embedding them into SiNx stacks. The most common measurement to resolve this question is the widely adopted variable stripe length (VSL) method where a 1D amplifier of variable length is created by means of a stripe excitation profile. This method, although easy to implement, has its pitfalls [62] especially in terms of collection of light for large stripe lengths when light starts to diffuse out of the acceptance cone of the collecting objective. A more robust approach is to use strip waveguides with varying length dictated by a deterministic etching process which support well defined optical modes that do not diffuse along the length of the amplifier. To this end, a $c a .50 \mathrm{~nm}$ spin coated layer of $\mathrm{CdSe} / \mathrm{CdS}$ flash QDs were integrated in a h-SiNx/QD/1-SiNx stack following the procedure outlined in section III. The planar stacks are then etched to form $5 \mu \mathrm{m}$ wide waveguides of varying lengths (see Fig. 9a,b).[63]

Characterization of light propagation at $900 \mathrm{~nm}$ (see Fig. 9c) allows us to evaluate the propagation loss for micron wide strips as only $\sim 1 \mathrm{~cm}^{-1}$. [44] As QDs have shown material gains up to $2.000 \mathrm{~cm}^{-1}$ (see Fig. 4c), we should be able to overcome the losses by optically exciting the QDs.

Pumping the waveguide stripes with the same pulsed light source as for the TAS measurements (section II) yields an exponentially increasing light output for waveguides 200-500 
$\mu \mathrm{m}$ long (see Fig. 9d). A threshold of fluence of $217 \mu \mathrm{J} / \mathrm{cm}^{2}$ compares quite well to the value of $140 \mu \mathrm{J} / \mathrm{cm}^{2}$ measured using TAS (see II.C). Fitting the distance-dependent light intensity to:

$$
I=C\left(\exp \left(g_{m} L\right)-1\right) / g_{m}
$$

yields a modal gain $g_{m}$ of $110 \pm 10 \mathrm{~cm}^{-1}$. These results clearly indicate that the exciting non-linear properties of QDs are transferable to embedded and hybrid QD/SiNx on-chip components. As was pointed out in IV, the modal gain can, neglecting dielectric effects in first approximation, also be written as a neat combination of the material gain $g_{i}$ (see II.C), the QD volume fraction in the film $f$ and the overlap between the optical mode and the QD layer $\Gamma$ :

$$
g_{m}=g_{i} \times f \times \Gamma
$$
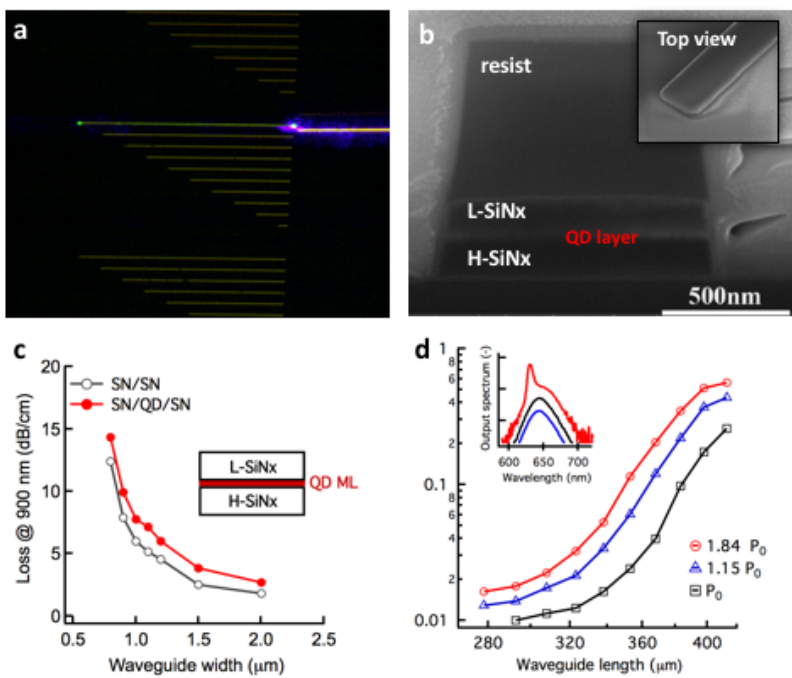

Fig. 9. Characterization of hybrid $\mathrm{SiNx} / \mathrm{QD}$ waveguides (a) Top view of a $\mathrm{SiNx} / \mathrm{QD}$ waveguide pumped with $400 \mathrm{~nm}$ where the facet emitted light is coupled to a lensed fiber for characterization (b) SEM image of the cross section of a H-SiNx/QD/L-SiNx stack. Note that the QD layer here is much thicker than the monolayer used in the measurements of $9 \mathrm{c}$, but compares to the $50 \mathrm{~nm}$ thick layer used in the amplifiation experiments shown in Fig. 9d. (c) Waveguide loss at $900 \mathrm{~nm}$ with (red) and without (black) a monolayer of quantum dots in between the $\mathrm{H}$ - and L-SiNx layers (see schematic inset) indicating that the presence of QDs does not increase the loss by much, a clear indication of the quality of the embedding process. (d) Light output from hybrid $5 \mu \mathrm{m}$ wide $\mathrm{SiNx} /(50 \mathrm{~nm}) \mathrm{QD} / \mathrm{SiNx}$ waveguides at different pump powers with different lengths under top pumping with a cylindrical lens and $110 \mathrm{fs}$ pulses at $400 \mathrm{~nm}$. A clear supralinear light output is observed which is characteristic of amplified spontaneous emission (ASE). Modal gain coefficients are extracted as 316 (black), 360 (blue) and $370 \mathrm{~cm}^{-1}$ (red). Note that $\mathrm{P}_{0}=2 \mathrm{P}_{\text {th }}$, with $\mathrm{P}_{\text {th }}$ the threshold power density for ASE.

Using the simulation tools outlined in section IV, we estimate $\Gamma$ to $23 \%$ and $f=0.45$. In combination with the material gain $g_{i}$ of ca. $1.000 \mathrm{~cm}^{-1}$ under the given pump intensity $\left(2 \mathrm{P}_{\text {th }}\right.$, see Fig. 4 d), the predicted modal gain of $\approx 100 \mathrm{~cm}^{-1}$ indeed comes very close to the measured values of $110 \mathrm{~cm}^{-1}$. Eventual discrepancies might arise from the reduced dielectric screening experienced by QDs in close-packed films leading to larger $g_{i}$ in thin films. In any case, the good correspondence obtained through eq. 7 confirms the idea that the solution based measurements put forward in section II are indeed appropriate for assessing QD material properties in hybrid SiNx stacks. Apart from providing a useful testbed for the effect of SiNx-embedding on the optical gain, the waveguides presented here show a first step towards QD-SiNx photonics and provide substantial net amplification to a well-defined guided mode, making them very useful for short - and long range on-chip optical interconnects. Moreover, the gain of $c a$. $100 \mathrm{~cm}^{-1}$ could suffice to provide net roundtrip gain in sufficiently qualitative nanophotonic resonators where the losses are typically in the range of $1-100 \mathrm{~cm}^{-1}$ (see section VI).

\section{DEMONSTRATOR}

We have arrived to the point where we can (a) embed and pattern SiNx/QD stacks with monolayer precision, (b) adequately model these hybrid stacks and (c) preserve and hence predict the exciting optical gain of the QDs after integration. The next step is to combine these efforts into fabricating a first on-chip demonstrator: an on-chip QDenabled micro-laser. As we aim for QDs to enable large scale and on-chip integrated photonics, we wanted the lasing cavity to be coupled to in-plane waveguides and to avoid complicated fabrication routines such as electron beam lithography. We therefore choose to fabricate micron-sided resonators (so-called 'microdisks') through classical optical lithography, coupled to a single mode bus waveguide for light extraction (see Figure 10a).[64], [65] The disks are freestanding and supported by a pillar of amorphous silicon. An important advantage of this design is that only the QD emission in the disk's whispering gallery modes couples to the bus waveguide, avoiding strong background spontaneous emission, a serious bottleneck in previous designs (see Fig. 10c, inset). [66] The coupling between the disk and the bus waveguide is a crucial addition to realize truly integrated light sources that couple their coherent radiation into guided modes of a PIC. Additionally, it offers a degree of freedom to tune the output efficiency of the disk laser. However, it does add a complexity to the design, in particular to the desired gain that the QD layer needs to provide.

\section{A. QDs as gain material in microresonators}

The proposed microcavity embeds QDs in a SiNx stack quite similar to the hybrid waveguides discussed earlier (see Figure 9). Mode simulations (see also section IV) indicate that in such a stack, maximum modal overlap is achieved for QDs positioned in the middle of the disk height, as is shown in Figure 10d. In section $\mathrm{V}$, we already demonstrated that the net modal gain of such guiding structures is $c a .100 \mathrm{~cm}^{-1}$ for a 55 $\mathrm{nm}$ thick film under optical pumping. Clearly, this puts a limit to the modal loss of the resonator as for lasing, it is exactly this loss that needs to be compensated. For microcavities, this loss $\alpha$ is typically expressed through a $\mathrm{Q}$ factor which connects to the effective loss coefficient $\alpha$ as: $Q=2 \pi n_{\text {eff }} / \lambda \alpha$, where $n_{\text {eff }}$ is the effective index of the disk mode at the wavelength interest. If we assume the maximum loss is given by the maximum achievable modal gain of $100 \mathrm{~cm}^{-1}$ in the gain band (see Figure 3) around $625 \mathrm{~nm}$, we obtain a minimal 
Q-factor of ca. 2000. Of course, one would aim for the highest Q-possible if only loss compensation was an issue, yet other factors determine the choice of Q such as outcoupling efficiency/power of the final device. If we want reasonable output, we attempt to be as close to this minimal Q - dictated by the QDs - as possible.
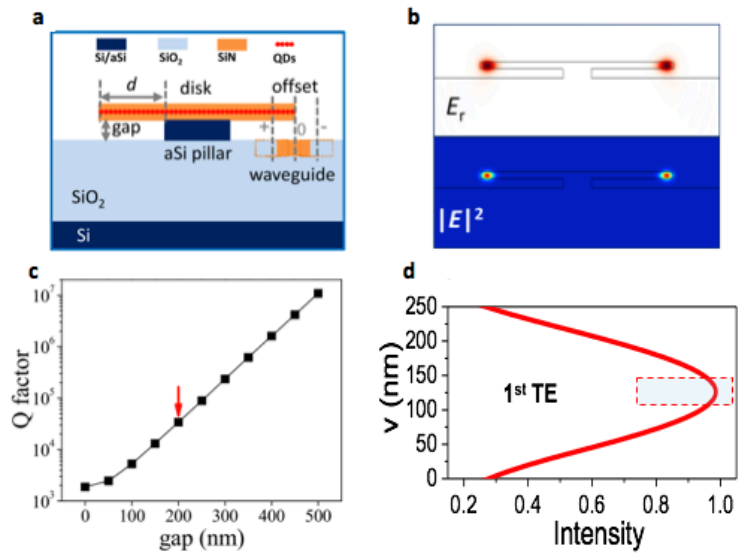

Fig. 10 Disk design and schematics (a) Cross-sectional schematic of SiNx disk with embedded QDs vertically coupled with an on- chip waveguide. (b) Simulated cross-sectional field profiles of radial component $\left(\mathrm{E}_{\mathrm{r}}\right)$ and intensity $\left(|E|^{2}\right)$ for the fundamental (quasi-)TE mode in suspended disk with a $7 \mu \mathrm{m}$ diameter. (c) Simulated Q factor (at a wavelength of $\sim 625 \mathrm{~nm}$ ) as a function of coupling gap for a $7 \mu \mathrm{m}$ diameter disk with $170 \mathrm{~nm}$ thickness (monolayer of QDs). The red arrow indicates the gap of $200 \mathrm{~nm}$ used in our devices. (d) Field intensity along the disk height of the fundamental TE mode. Note that the height is adjusted to include the $55 \mathrm{~nm}$ thick QD film used in the final device. The dashed box indicates the position of the $c a .55 \mathrm{~nm}$ thick QD film in the final device. Adapted from ref [65]. Copyright 2015 OSA

\section{B. Design and linear characterization}

The design of the desired high Q resonator relies on FDTD simulations, where a dispersive refractive index for the QDs is used (see section IV and Figure 7a). The Q-factor of the micro-resonator is linked to the losses of the system: outcoupling to the bus waveguide $\left(Q_{c}\right.$, which is a necessary loss to extract the light), the scattering loss $\left(Q_{\text {scat }}\right.$, scattering throughsidewall roughness, limited by fabrication) and intrinsic radiative loss $\mathrm{Q}_{\mathrm{r}}$ :

$$
\frac{1}{Q}=\frac{1}{Q_{c}}+\frac{1}{Q_{s c a t}}+\frac{1}{Q_{r}}
$$

The parameters of the system are chosen as follows:

- Disk diameter: Mainly affects the scattering loss $Q_{\text {scat }}$ as smaller disks push the modes more to the outer surface. See also Figure 11d. The scattering losses are minimized through the procedures outlined in section III.

- Disk height: $250 \mathrm{~nm}$, based on the requirement to have whispering gallery modes at $625 \mathrm{~nm}$ with low radiative loss (high $\mathrm{Q}_{\mathrm{r}}$ ).

- Waveguide height: $125 \mathrm{~nm}$, corresponding to a fundamental TE mode at $625 \mathrm{~nm}$.
- Gap distance: Strongly affects the radiatively limited $Q_{r}$ (see Figure 10c) and the coupling to the bus waveguide. A gap distance of $300 \mathrm{~nm}$ is chosen.

The main parameters to tune the coupling $Q_{c}$, see also previous work by Xie et al. [65]:

- Waveguide width \& offset distance: The effect of both can be used to tune the coupling efficiency from 0.1 to $5 \%$.

Simulations indicate that an impressive $\mathrm{Q}_{\mathrm{r}}$ of up to 1 million for quasi-TE modes can be reached (see Figure 10c). Although this is very promising, this will clearly be reduced due to fabrication imperfections and required outcoupling for an actual device.

To evaluate the disk fabrication, we focused initially on disks with a monolayer of QDs embedded to study the disk as such. Using top-down excitation, we can photo-excite the embeddd QD layer and the emission is collected from the bus waveguide. As is shown in Figure $\mathrm{X}$ (taken from Weiqiang et $a l$. [65]), we can easily attain loaded Q-factors of up to 5000, surpassing the required $\mathrm{Q} \cong 2000$ with ease. These results indicate that the loaded disks should be able to lase, given that we increase the QD layer thickness from the monolayer regime to the ca. $55 \mathrm{~nm}$ thickness that provides the gain required to compensate the $\mathrm{Q}$ of $c a .2000$.
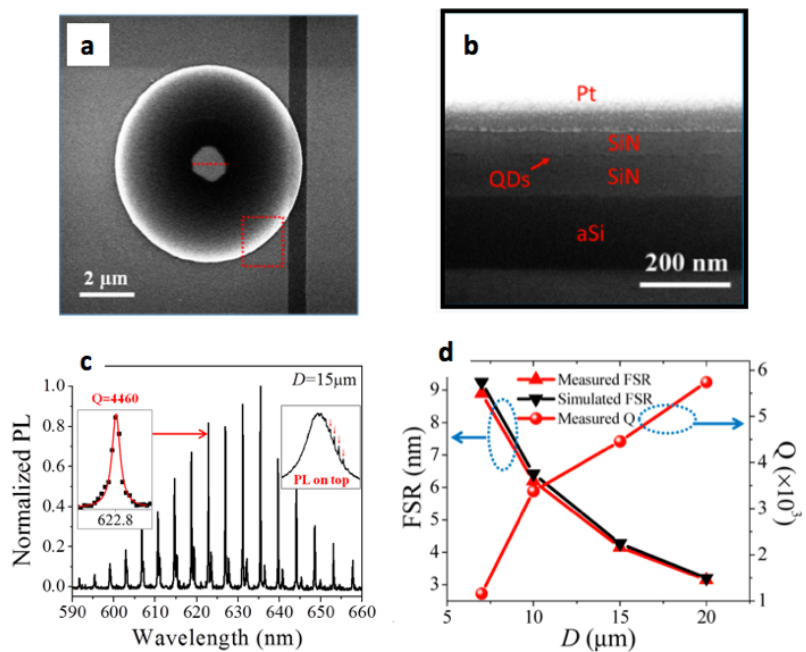

Fig. 11 Disk fabrication and linear characterization of disks with a single monolayer of flash CdSe/Cds QDs embedded. (a) Top view of a $7 \mu \mathrm{m}$ disk with bus waveguide with $\sim 500 \mathrm{~nm}$ waveguide width and $-160 \mathrm{~nm}$ offset (b) Focused Ion Beam (FIB) cross section of disk of (a) along dashed red line. (c) Background-free spectrum of the emitted light under low-fluence excitation collected at the output facet of the bus waveguide and (inset) broadened PL spectrum collected from the top of a $15 \mu \mathrm{m}$ disk using a confocal collection. A Q-factor of 4460 is extracted for the fundamental TE WGM mode at 622.8 $\mathrm{nm}$. (d) Measured and simulated free spectral range (FSR) values and fitted Q factors for different diameter (D) disks with a monolayer of QDs. Both the FSR and Q are calculated for the modes around $\sim 622 \mathrm{~nm}$, and Q factors are measured for the devices with $\sim 500 \mathrm{~nm}$ waveguide width and $-160 \mathrm{~nm}$ offset. Adapted from ref [65]. Copyright 2015 OSA

\section{Results}

Fig. 11 shows an overview of the results obtained on an $7 \mu \mathrm{m}$ microdisk $(\mathrm{Q} \cong 2000)$ under top-down picosecond pulsed excitation at $400 \mathrm{~nm}$ where the light is collected from the bus 
waveguide. A clear supralinear increase of the light emitted into the specific cavity modes is observed, together with a shortening of the lifetime of the QD emission as it transitions from spontaneous emission to stimulated emission, yet another characteristic of lasing. Fig. 12d shows that the light emitted by the QDs couples to the whispering gallery mode when lasing action kicks in. [51][64] We like to stress that we show results for the $7 \mu \mathrm{m}$ disk as this is the most challenging due to the low Q. Xie et al.[64] have shown that also larger disks lase which is not entirely unexpected due to the higher Q-factor (see Figure 11d). Although the results presented here are not the first demonstration of QD lasing[67]-[69], nor a demonstration with the lowest threshold [70], it is the first fully integrated on-chip colloidal QD laser, thereby clearly demonstrating the massive potential for colloidal QDs to enable cost-effective and more versatile next-generation integrated photonic circuits. Several optimizations of the exisisting design are ongoing, in particular related to both the QD gain material (various core/shell combinations) and QD layer thickness in the active device. Further optimizaton of the etching protocol could also lead to lasing in smaller disks, hence obtaining larger FSRs.
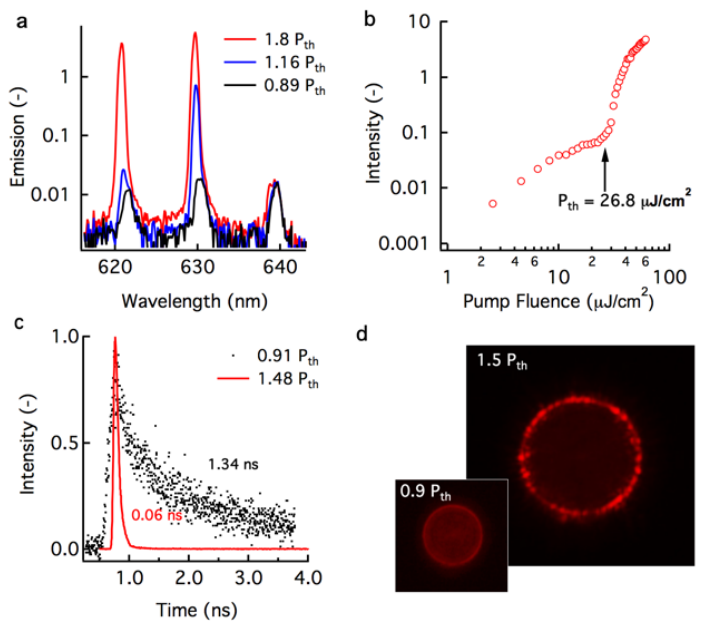

Fig. 12. Overview of on-chip microdisk laser ( $7 \mu \mathrm{m}$ diameter, gap $300 \mathrm{~nm}$, offset $200 \mathrm{~nm}$ ) with a $55 \mathrm{~nm}$ QD gain layer (total disk height of $250 \mathrm{~nm}$ ) under top-down picosecond pulsed excitation at $450 \mathrm{~nm}$. (a) spectra below and above threshold $P_{\text {th }}(\mathrm{b})$ Integrated intensity versus pump power, showing a clear S-curve with a threshold of $26.8 \mu \mathrm{J} / \mathrm{cm}^{2}$. (c) Output intensity versus time below (black markers) and above threshold (red line) and (d) optical microscope image of a $15 \mu \mathrm{m}$ disk above and below threshold showing the transition from spontaneous emission to stimulated emission into a single whispering gallery mode. [51]

\section{CONCLUSION AND OUTLOOK}

We have presented a design flow for hybrid QD-silicon nitride components where starting from colloidal dispersions various integrated components were demonstrated, from simple strip waveguide amplifiers to fully integrated on-chip lasers. However, the toolbox clearly allows us to explore a variety of new routes for hybrid QD-SiNx optoelectronics.

\section{A. Continuous Wave Pumping}

A long sought after breakthrough for QD opto-electronics is a continuous-wave (CW) laser, pumped either optically at first but preferably electrically driven in the future.[71] Realistic current densities in state-of-the-art direct current QD-LEDs are in the order of several $\mathrm{A} / \mathrm{cm}^{2}([72]-[74])$ implying that optical pumping thresholds should drop to the order of a few $\mathrm{W} / \mathrm{cm}^{2}$. The only report so-far on ASE and lasing under these extremely low thresholds is that of Grim et al. [70] who reported ASE at a threshold of $6 \mathrm{~W} / \mathrm{cm}^{2}$ using quasi-2D CdSe platelets. Although this result is under debate, it clearly points towards the potential of QD based gain media to reach such low threshold densities in the near future. Apart from this sole report, a more common threshold for lasing is that of several tens of $\mathrm{kW} / \mathrm{cm}^{2}$ observed by Adachi and Fan et al.[75],[76] who reached these low thresholds by focusing on the thermal conductivity and packing density of their QD films through ligand exchange and a focus on the excited state lifetime, i.e. the timescale on which population inversion can be sustained. Although a direct comparison between thresholds under pulsed operation and continuous wave are often difficult, we believe that the microdisk laser presented in the previous section can be pushed to $\mathrm{CW}$ operation by improving on the points mentioned by Fan et al. [76], especially since the QDs used in that seminal work show properties (such as gain lifetime, cross section, ..) comparable to the 'flash' QDs used here. Although the threshold densites remain quite high for comparable electrical excitation, we are strongly convinced there are many applications for optically pumped integrated SiN-QD devices. As a matter of fact some of the most successful optical devices in use today are optically pumped (e.g. Erbium Doped Fiber Amplifiers, Nd:YAG-lasers, tunable TiSaph lasers ...). Typically, this is relevant if "low quality" pump light (i.e. light with low coherence, light with low beam quality ...) is translated in "high quality" light (single wavelength lasing, lasing with high beam quality, tunable lasing, amplification of WDM signals, ...). Although nitride seems to rule out electrical excitation, it was shown by Bozyigit et al. [77] that field driven ionization in insulator/QD stacks can lead to electroluminescence.
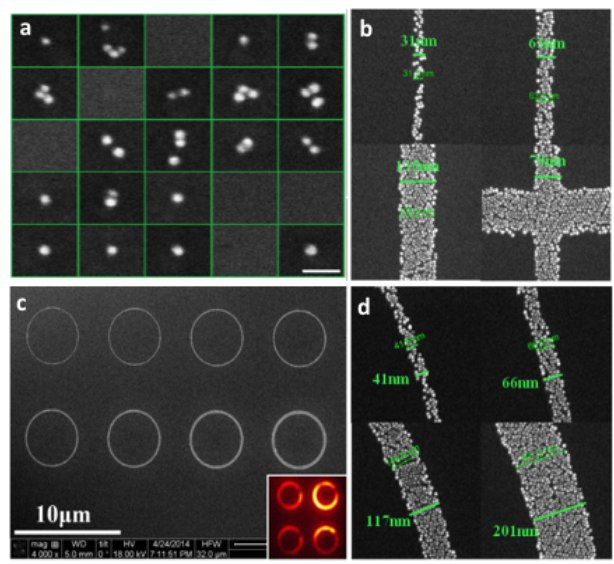

Fig. 13. SEM images of the nanoscale patterns for QD films. (a) SEM image of $5 \times 5$ dot patterns of QDs by using the resist hole of $31.6 \mathrm{~nm}$ diameter and $\sim 33 \mathrm{~nm}$ thickness. All images have the same scale bar of $50 \mathrm{~nm}$ as shown in 
the image at the right bottom corner. (b) Line and (c) ring patterns with different widths, and the enlarged views for some selected widths for line ring (d) patterns. The inset at the right lower corner in (c) shows the respective micro-PL of the corresponding pattern shapes. Reprinted with permission from ref. [78]. Copyright 2015 American Chemical Society.

\section{B. Patterning}

Deterministic patterning of QD films is extremely relevant for either (a) single QD deposition and (b) improving light-matter interaction. Indeed, when the QDs could be deposited where the field intensity of the fundamental mode is highest, modal gain would improve drastically reducing the thresholds for stimulated emission and lasing. For example, the ring patterns in Fig. 13 would match perfectly to the doughnut-shaped mode profiles of the fundamental whipsering gallery modes of the microdisk laser (see Figure 10). It is expected that also the etching process (and hence the scattering losses) would benefit as we no longer have to etch through a thick QD layer. Single dot patterning on the other hand is a must for future singlephoton sources (see section VI.C). Using an optimized lift-off process we are able to achieve both nanoscale and single-dot patterning (see Fig. 13a) allowing us to locally deposit QDs in the near future.

\section{Single Photon Sources}

The application potential of integrated light sources is not only limited to the classical applications such as light emitting diodes or lasers. All-optical quantum information processing has shown vast potential in areas as computing, cryptography and random number generation. [79], [80] Colloidal QDs are prime candidates for realizing on-chip single photon sources due to the two-level nature of their emitting states. Indeed, numerous reports indicate the potential of QD, in particular even for the flash-type CdSe/CdS QDs used in this work[81], for this purpose, yet no clear road towards integration is presented mainly due to the low coupling of QD light emission into nanophotonic structures. Even so, the use of onchip light sources could be greatly beneficial due to the larger scale on which computations could be carried out, often required for error compensation, and the cost of quantum photonic components could be drastically reduced. The work of Bisschop et al.[82] demonstrates that high coupling efficiency and polarization contrast can be attained in the relatively simple silicon nitride waveguides used here. The ability to pattern single dots (see Figure 13a) and fabricate high Q cavities (see Figure 10) coupled direclty to PICs, the silicon nitride platform presented here clearly offers a road towards on-chip QD-based quantum optics.

\section{Improved mode coupling}

For the 1D amplifiers outlined in section V, a considerable fraction of the initial spontaneous emission will not couple to the guided mode that provides amplification, which will increase e.g. the gain threshold. Typical values for the mode confinement in the active layer of $\mathrm{SiNx}$ waveguides with 2D layers of QDs are $c a .20 \%$, which is an improvement over the simple 'coated' waveguides of section V.A where only a few percent of the guided mode was found in the QD layer. When considering emission of only a few QDs, for example for single photon sources (see previous), this is even more relevant as the collection efficiency of single photons is key to many applications. It is therefore instructive to attempt improvements of the coupling efficiency and consider alternatives to the simple strip waveguide on oxide. Embedding the QDs directly into the bulk of the component drastically improved the coupling efficiency to e.g. the fundamental mode of a straight waveguide, but Bisschop et al. [82] indicated that by defining slot waveguides or suspended strip waveguides, the coupling efficiency for spontaneous emission can be as high as $\sim 70 \%$. A strong polarization dependence was observed despite the small index contrast between the QDs and SiNx, which in turn could be useful for engineering the polarization state of single photons (see section VI.C).

\section{ACKNOWLEDGMENTS}

P.G. acknowledges the Flemish Research Fund (FWO) for a post-doctoral fellowship. D.V.T and W.Xie acknowledge the ERC-ULPPIC, H2020-MSCA Phonsi and the IAP Photonics@be projects for financial support. Z.H. acknowledges support by the European Comission via the Marie-Sklodowska Curie action Phonsi (H2020-MSCA-ITN642656), the Belgian Science Policy Office (IAP 7.35, photonics@be), IWT-Vlaanderen (SBO-MIRIS) and Ghent University (GOA no. 01G01513) for funding. T. Stöferle and R. Mahrt (IBM Research Zürich) are acknowledged for their help with the characterization of the on-chip disk laser.

\section{REFERENCES}

[1] A. Rickman, "The commercialization of silicon photonics," Nat. Photonics, vol. 8, no. 8, pp. 579-582, Jul. 2014.

A. Z. Subramanian, E. Ryckeboer, A. Dhakal, F. Peyskens, A. Malik, B. Kuyken, H. Zhao, S. Pathak, A. Ruocco, A. De Groote, P. Wuytens, D. Martens, F. Leo, W. Xie, U. D. Dave, M. Muneeb, P. Van Dorpe, J. Van Campenhout, W. Bogaerts, P. Bienstman, N. Le Thomas, D. Van Thourhout, Z. Hens, G. Roelkens, and R. Baets, "Silicon and silicon nitride photonic circuits for spectroscopic sensing on-achip," Photonics Res., vol. 3, no. 5, p. B47, 2015.

[3] A. Jenkins, "Silicon lasers: the final frontier," Nat. Photonics, vol. 1, no. 240, 2007.

H. Rong, R. Jones, L. Ansheng, O. Cohen, D. Hak, A. Fang, and M. Paniccia, "A continuous-wave Raman silicon laser," Nature, vol. 433, no. 7027, pp. 717-9, Feb. 2005.

L. Pavesi, L. Dal Negro, C. Mazzoleni, G. Franzò, and F. Priolo, "Optical gain in silicon nanocrystals.," Nature, vol. 408, no. 6811, pp. 440-4, Nov. 2000.

A. Dhakal, P. C. Wuytens, K. Jans, N. Le Thomas, and R. Baets, "Nanophotonic Waveguide Enhanced Raman Spectroscopy of Biological Submonolayers," ACS Photonics, vol. 3, pp. 2141-2149, 2016.
R. Roelkens, G. Liu, L. Liang, D. Jones, A. Fang, B. Koch, 
and J. Bowers, "III-V/Silicon Photonics for On-Chip and Inter-Chip Optical Interconnects," Laser Photon. Rev., vol. 4, no. 6, pp. 751-779, 2010.

Z. Wang, B. Tian, M. Pantouvaki, W. Guo, P. Absil, J. Van Campenhout, C. Merckling, and D. Van Thourhout, "Room Temperature InP DFB Laser Array Directly Grown on (001) Silicon," Nat. Photonics, vol. 9, no. 12, pp. 837-842, 2015.

S. Chen, W. Li, J. Wu, Q. Jiang, M. Tang, S. Shutts, S. N. Elliott, A. Sobiesierski, A. J. Seeds, I. Ross, P. M. Smowton, and H. Liu, "Electrically pumped continuous-wave III - V quantum dot lasers on silicon," Nat. Photonics, vol. 10, no. 5, pp. 307-311, 2016.

[10] C. R. Kagan, E. Lifshitz, E. H. Sargent, and D. V. Talapin, "Building devices from colloidal quantum dots," Science (80-. )., vol. 353, no. 6302, 2016.

[11] M. V Kovalenko, L. Manna, A. Cabot, Z. Hens, D. V Talapin, C. R. Kagan, V. I. Klimov, A. L. Rogach, P. Reiss, D. J. Milliron, P. Guyot-sionnnest, G. Konstantatos, W. J. Parak, T. Hyeon, B. A. Korgel, C. B. Murray, and W. Heiss, "Prospects of nanoscience with nanocrystals," ACS Nano, vol. 9, no. 2, pp. 1012-1057, 2015.

[12] Y. Yin and P. Alivisatos, "Colloidal nanocrystal synthesis and the organic-inorganic interface.," Nature, vol. 437, no. 7059, pp. 664-70, Sep. 2005.

[13] F. Meinardi, H. Mcdaniel, F. Carulli, A. Colombo, K. A. Velizhanin, N. S. Makarov, R. Simonutti, V. I. Klimov, and S. Brovelli, "Highly efficient large-area colourless luminescent solar concentrators using heavy-metal-free colloidal quantum dots," Nat. Nanotechnol., vol. 10, no. 10, pp. 878-885, 2015.

[14] O. Benson, "Assembly of hybrid photonic architectures from nanophotonic constituents," Nature, vol. 480, no. 7376, pp. 193-199, 2011.

[15] R. Bose, J. Gao, J. F. McMillan, A. D. Williams, and C. W. Wong, "Cryogenic spectroscopy of ultra-low density colloidal lead chalcogenide quantum dots on chip-scale optical cavities towards single quantum dot near-infrared cavity QED.," Opt. Express, vol. 17, no. 25, pp. 22474-83, 2009.

[16] R. Bose, X. Yang, R. Chatterjee, J. Gao, and C. W. Wong, "Weak coupling interactions of colloidal lead sulphide nanocrystals with silicon photonic crystal nanocavities near $1.55 \mu \mathrm{m}$ at room temperature," Appl. Phys. Lett., vol. 90, no. 11, pp. 1-4, 2007.

[17] M. Humer, R. Guider, W. Jantsch, and T. Fromherz, "Integration, photostability and spontaneous emission rate enhancement of colloidal PbS nanocrystals for Si-based photonics at telecom wavelengths," Opt. Express, vol. 21, no. 16, pp. 18680-18688, 2013.

[18] J. C. Weeber, K. Hammani, G. Colas-Des-Francs, A. Bouhelier, J. Arocas, A. Kumar, F. Eloi, S. Buil, X. Qu??lin, J. P. Hermier, M. Nasilowski, and B. Dubertret, "Colloidal
Quantum Dot Integrated Light Sources for Plasmon Mediated Photonic Waveguide Excitation," ACS Photonics, vol. 3, no. 5, pp. 844-852, 2016.

[19] Z. Wu, Z. Mi, P. Bhattacharya, T. Zhu, and J. Xu, "Enhanced spontaneous emission at 1.55 um from colloidal $\mathrm{PbSe}$ quantum dots in a Si photonic crystal microcavity," Appl. Phys. Lett., vol. 90, no. 17, pp. 1-4, 2007.

[20] E. Drijvers, J. De Roo, P. Geiregat, K. Feher, Z. Hens, and T. Aubert, "Revisited Wurtzite CdSe Synthesis: A Gateway for the Versatile Flash Synthesis of Multishell Quantum Dots and Rods," Chem. Mater., vol. 28, no. 20, pp. 73117323, 2016 .

[21] S. Ithurria, M. D. Tessier, B. Mahler, R. P. S. M. Lobo, B. Dubertret, and A. L. Efros, "Colloidal nanoplatelets with two-dimensional electronic structure," Nat. Mater., vol. 10, no. 10, pp. 1-6, Oct. 2011.

[22] E. Izquierdo, A. Robin, N. Lequeux, and E. Lhuillier, "Strongly confined $\mathrm{HgTe} 2 \mathrm{D}$ nanoplatelets as narrow near infrared emitter," vol. 30, no. 2015, p. 2015.

[23] M. D. Tessier, D. Dupont, and Z. Hens, "InP / ZnS and InP / ZnSe Colloidal Quantum Dots ."

M. Cirillo, T. Aubert, R. Gomes, R. Van Deun, P. Emplit, A Biermann, H. Lange, C. Thomsen, E. Brainis, and Z. Hens, " Flash' Synthesis of CdSe/CdS Core - Shell Quantum Dots," Chem. Mater., vol. 26, pp. 1154-1160, 2014.

[25] A. L. Efros, "Non-blinking Semiconductor Nanocrystals : Suppression of Nonradiative Auger Processes," no. June, 2009.

[26] G. E. Cragg and A. L. Efros, "Suppression of Auger processes in confined structures.," Nano Lett., vol. 10, no. 1, pp. 313-7, Jan. 2010.

[27] F. García-Santamaría, S. Brovelli, R. Viswanatha, J. A. Hollingsworth, H. Htoon, S. A. Crooker, and V. I. Klimov, "Breakdown of Volume Scaling in Auger Recombination in CdSe / CdS Heteronanocrystals : The Role of the Core-Shell Interface," Nano Lett., 2011.

[28] A. Houtepen, Z. Hens, J. S. Owen, and I. Infante, "On the Origin of Surface Traps in Colloidal II-VI Semiconductor Nanocrystals," Chem. Mater., vol. Just Accep, 2016.

[29] Z. Hens and I. Moreels, "Light Absorption by Colloidal Semiconductor Quantum Dots," J. Mater. Chem., vol. 22, pp. 10406-10415, 2012.

[30] A. W. Achtstein, A. Antanovich, A. Prudnikau, R. Scott, U. Woggon, and M. Artemyev, "Linear Absorption in CdSe Nanoplates: Thickness and Lateral Size Dependency of the Intrinsic Absorption," J. Phys. Chem. C, vol. 119, no. 34, pp. 20156-20161, 2015.

[31] I. Moreels, K. Lambert, D. Smeets, D. De Muynck, T. Nollet, J. C. Martins, F. Vanhaecke, A. Vantomme, C. Delerue, G. Allan, and Z. Hens, "Size-dependent optical 
properties of colloidal $\mathrm{PbS}$ quantum dots.," ACS Nano, vol. 3, no. 10, pp. 3023-30, Oct. 2009.

[32] B. De Geyter and Z. Hens, "The absorption coefficient of $\mathrm{PbSe} / \mathrm{CdSe}$ core/shell colloidal quantum dots," Appl. Phys. Lett., vol. 97, no. 16, p. 161908, 2010.

[33] I. Angeloni, W. Raja, R. Brescia, A. Polovitsyn, and F. De Donato, "Disentangling the Role of Shape, Ligands, and Dielectric Constants in the Absorption Properties of Colloidal CdSe/CdS Nanocrystals," ACS Photonics, vol. 3, pp. 58-67, 2016.

[34] S. Adachi, Optical Constants of Crystalline and Amorphous Semiconductors. Springer Science, 1999.

[35] P. Geiregat, Y. Justo, S. Abé, S. Flamee, and Z. Hens, "Giant and Broadband Absorption Enhancement in Colloidal Quantum Dot Monolayers through Dipolar Coupling," ACS Nano, vol. 7, no. 2, pp. 987-993, Jan. 2013.

[36] V. I. Klimov, A. A. Mikhailovsky, S. Xu, A. Malko, J. A. Hollingsworth, C. A. Leatherdale, H.-J. Eisler, and M. G. Bawendi, "Optical gain and stimulated emission in nanocrystal quantum dots," Science (80-. )., vol. 290, no. 5490, pp. 314-317, Oct. 2000.

[37] V. Klimov, "Optical Nonlinearities and Ultrafast Carrier Dynamics in Semiconductor Nanocrystals," Phys. Chem. B, vol. 104, no. 26, pp. 6112-6123, Jul. 2000.

[38] A. G. Midgett, J. M. Luther, J. T. Stewart, D. K. Smith, L. A. Padilha, V. I. Klimov, A. J. Nozik, and M. C. Beard, "Size and Composition Dependent Multiple Exciton Generation E ffi ciency in PbS, PbSe, and PbS," Nano Lett., vol. 13, no. 2, p. 3078-3085, 2013.

[39] M. Pietryga, Y. Park, J. Lim, A. F. Fidler, W. K. Bae, S. Brovelli, and V. I. Klimov, "Spectroscopic and Device Aspects of Nanocrystal Quantum Dots," Chem. Rev., 2016.

[40] F. García-Santamaría, Y. Chen, J. Vela, R. D. Schaller, J. a Hollingsworth, and V. I. Klimov, "Suppressed auger recombination in 'giant' nanocrystals boosts optical gain performance.," Nano Lett., vol. 9, no. 10, pp. 3482-8, Oct. 2009.

[41] V. I. Klimov, S. a Ivanov, J. Nanda, M. Achermann, I. Bezel, J. a McGuire, and A. Piryatinski, "Single-exciton optical gain in semiconductor nanocrystals.," Nature, vol. 447, no. 7143, pp. 441-6, May 2007.

[42] N. C. Anderson, M. P. Hendricks, J. J. Choi, and J. S. Owen, "Ligand exchange and the stoichiometry of metal chalcogenide nanocrystals: Spectroscopic observation of facile metal-carboxylate displacement and binding," J. Am. Chem. Soc., vol. 135, no. 49, pp. 18536-18548, 2013.

[43] V. I. Klimov, "Spectral and dynamical properties of multiexcitons in semiconductor nanocrystals.," Annu. Rev. Phys. Chem., vol. 58, pp. 635-73, Jan. 2007.
Van Thourhout, "Low-loss silicon nitride waveguide hybridly integrated with colloidal quantum dots," Opt. Express, vol. 23, no. 9, pp. 12152-12160, 2015.

[45] T. D. Visser, H. Blok, B. Demeulenaere, and D. Lenstra, "Confinement factors and gain in optical amplifiers," IEEE J. Quantum Electron., vol. 33, no. 10, pp. 1763-1766, 1997.

[46] K. Lambert, R. K. Capek, M. I. Bodnarchuk, M. V Kovalenko, D. Van Thourhout, W. Heiss, and Z. Hens, "Langmuir-Schaefer deposition of quantum dot multilayers.," Langmuir, vol. 26, no. 11, pp. 7732-6, Jun. 2010.

[47] K. Lambert, R. K. Capek, M. I. Bodnarchuk, M. V Kovalenko, D. Van Thourhout, W. Heiss, and Z. Hens, "Langmuir-Schaefer deposition of quantum dot multilayers.," Langmuir, vol. 26, no. 11, pp. 7732-6, Jun. 2010.

[48] A. Omari, W. Xie, P. Geiregat, D. Van Thourhout, and Z. Hens, "Modeling the Optical Properties of Low-cost Colloidal Quantum Dot Functionalized Strip SOI Waveguides," IEEE J. Sel. Top. Quantum Electron., vol. 20, no. AUGUST, pp. 1-1, 2014.

[49] A. Omari, P. Geiregat, D. Van Thourhout, and Z. Hens, "Assessing Colloidal Quantum Dot Optical Properties through Free Standing Layers on Photonic Waveguides," in E-MRS 2013 Spring \& Bilateral, 2013.

[50] W. Xie, R. Gomes, T. Aubert, S. Bisschop, Y. Zhu, Z. Hens, E. Brainis, and D. Van Thourhout, "Nanoscale and SingleDot Patterning of Colloidal Quantum Dots," Nano Lett., p. $151020091920006,2015$.

W. Xie, T. Stöferle, G. Rainò, T. Aubert, Y. Zhu, R. F. Mahrt, Z. Hens, and D. Van Thourhout, "Integrated Silicon Nitride Microdisk Lasers Based on Quantum Dots," in CLEO:2016, 2016, vol. JTh4B.6.

[52] Z. Hens and I. Moreels, "Light absorption by colloidal semiconductor quantum dots," J. Mater. Chem., vol. 22, no. 21, p. 10406, 2012.

[53] K. Devloo-casier, P. Geiregat, K. F. Ludwig, K. Van Stiphout, A. Vantomme, Z. Hens, C. Detavernier, and J. Dendooven, "A Case Study of ALD Encapsulation of Quantum Dots: Embedding Supported CdSe/CdS/ZnS Quantum Dots in a ZnO Matrix," 2016.

[54] I. Moreels, G. Allan, B. De Geyter, L. Wirtz, C. Delerue, and Z. Hens, "Dielectric function of colloidal lead chalcogenide quantum dots obtained by a Kramers-Krönig analysis of the absorbance spectrum," Phys. Rev. B, vol. 81, no. 23, pp. 1-7, Jun. 2010.

[55] B. T. Diroll, E. A. Gaulding, C. R. Kagan, and C. B. Murray, "Spectrally-Resolved Dielectric Functions of Solution-Cast Quantum Dot Thin Films," Chem. Mater., p.

$150828144815008,2015$.
T. D. Visser, B. Demeulenaere, J. Haes, D. Lenstra, R. 
Baets, and H. Blok, "Confinement and modal gain in dielectric waveguides," J. Light. Technol., vol. 14, no. 5, pp. 885-887, 1996.

[57] C. Kagan, C. Murray, M. Nirmal, and M. Bawendi, "Electronic energy transfer in CdSe quantum dot solids.," Phys. Rev. Lett., vol. 76, no. 9, pp. 1517-1520, 1996.

[58] A. Omari, P. Geiregat, D. Van Thourhout, and Z. Hens, "Light Absorption in Hybrid Silicon-on-Insulator/Quantum Dot Waveguides," Opt. Express, vol. 21, no. 20, p. 23272 , Sep. 2013.

[59] Y. Justo, P. Geiregat, K. Van Hoecke, F. Vanhaecke, C. D. M. Donega, and Z. Hens, "Optical Properties of PbS / CdS Core / Shell Quantum Dots," J. Phys. Chem. C, vol. 117, no. 39, pp. 20171-20177, 2013.

[60] A. Omari, P. Geiregat, D. Van, and Z. Hens, "Light absorption in hybrid silicon-on-insulator / quantum dot waveguides," no. 2006, pp. 933-934, 2012.

[61] A. Omari, W. Xie, P. Geiregat, and D. Van, "Modeling the optical properties of low-cost colloidal quantum dot functionalized strip SOI waveguides," J. Sel. Top. Quantum Electron., vol. XX, no. Xx, pp. 1-6, 2013.

[62] L. Negro, P. Bettotti, and L. Pavesi, "Applicability conditions and experimental analysis of the variable stripe length method for gain measurements," Opt. Commun., vol. 229, no. 1-6, pp. 337-348, Jan. 2004.

[63] Y. Zhu, W. Xie, P. Geiregat, S. Bisschop, and T. Aubert, "Hybrid Colloidal Quantum Dot Silicon Nitride Waveguide Gain Measurement Based on Variable Stripe Length Method," in Cleo 2016, 2016, no. ATh1J.5.

[64] W. Xie, T. Stöferle, G. Raino, T. Aubert, S. Bisschop, Y. Zhu, R. F. Mahrt, P. Geiregat, E. Brainis, Z. Hens, and D. Van Thourhout, "On-Chip Integrated Quantum-Dot SiliconNitride Microdisk Lasers,” Adv. Mater., p. 1604866, 2017.

[65] W. Xie, Y. Zhu, T. Aubert, Z. Hens, E. Brainis, and D. Van Thourhout, "Fabrication and characterization of on-chip silicon nitride microdisk integrated with colloidal quantum dots," Opt. Express, vol. 24, no. 2, pp. 114-122, 2016.

[66] B. De Geyter, K. Komorowska, E. Brainis, P. Emplit, P. Geiregat, A. Hassinen, Z. Hens, and D. Van Thourhout, "From Fabrication to Mode Mapping in Silicon Nitride Microdisks with Embedded Colloidal Quantum Dots," Appl. Phys. Lett., vol. 101, no. 16, p. 161101, 2012.

[67] B. Guzelturk, Y. Kelestemur, K. Gungor, A. Yeltik, M. Z. Akgul, Y. Wang, R. Chen, C. Dang, H. Sun, and H. V. Demir, "Stable and Low-Threshold Optical Gain in CdSe/CdS Quantum Dots: An All-Colloidal Frequency UpConverted Laser," Adv. Mater., vol. 27, no. 17, pp. 27412746, 2015.

[68] C. Dang, J. Lee, C. Breen, J. S. Steckel, S. Coe-Sullivan, and A. Nurmikko, "Red, green and blue lasing enabled by single-exciton gain in colloidal quantum dot films," Nat.
Nanotechnol., vol. 7, no. 5, pp. 335-339, Apr. 2012.

[69] C. Grivas, C. Li, P. Andreakou, P. Wang, M. Ding, G. Brambilla, L. Manna, and P. Lagoudakis, "Single-mode tunable laser emission in the single-exciton regime from colloidal nanocrystals," Nat. Commun., vol. 4, p. 2376, Aug. 2013.

[70] J. Q. Grim, S. Christodoulou, F. Di Stasio, R. Krahne, R. Cingolani, L. Manna, and I. Moreels, "Continuous-wave biexciton lasing at room temperature using solutionprocessed quantum wells.," Nat. Nanotechnol., vol. 9, pp. 891-895, Oct. 2014.

[71] A. Nurmikko, "What future for quantum dot-based light emitters?," Nat. Nanotechnol., vol. 10, no. 12, pp. 10011004, 2015.

[72] J. M. Caruge, J. E. Halpert, V. Wood, V. Bulović, and M. G. Bawendi, "Colloidal quantum-dot light-emitting diodes with metal-oxide charge transport layers," Nat. Photonics, vol. 2, no. 4, pp. 247-250, Mar. 2008.

[73] X. Dai, Z. Zhang, Y. Jin, Y. Niu, H. Cao, X. Liang, L. Chen, J. Wang, and X. Peng, "Solution-processed, highperformance light-emitting diodes based on quantum dots," Nature, vol. 515, no. 7525, pp. 96-99, 2014.

[74] A. L. Rogach, N. Gaponik, J. M. Lupton, C. Bertoni, D. E. Gallardo, S. Dunn, N. L. Pira, M. Paderi, P. Repetto, S. G. Romanov, C. O’Dwyer, C. M. S. Torres, and A. Eychmuller, "Light-emitting diodes with semiconductor nanocrystals," Angew. Chemie-international Ed., vol. 47, no. 35, pp. 65386549, 2008.

[75] M. M. Adachi, F. Fan, D. P. Sellan, S. Hoogland, O. Voznyy, A. J. Houtepen, K. D. Parrish, P. Kanjanaboos, J. A. Malen, and E. H. Sargent, "Microsecond-sustained lasing from colloidal quantum dot solids," Nat. Commun., vol. 6, p. 8694, 2015.

[76] F. Fan, O. Voznyy, P. Randy, K. T. Bicanic, M. M. Adachi, J. R. Mcbride, K. R. Reid, Y. Park, X. Li, A. Jain, R. Quintero-bermudez, M. Saravanapavanantham, M. Liu, M. Korkusinski, P. Hawrylak, V. I. Klimov, S. J. Rosenthal, S. Hoogland, and E. H. Sargent, "Continuous-wave lasing in colloical quantum dot solids enabled by facet-selective epitaxy," Nature, vol. Advance On, 2017.

D. Bozyigit, V. Wood, Y. Shirasaki, and V. Bulovic, "Study of field driven electroluminescence in colloidal quantum dot solids," J. Appl. Phys., vol. 111, no. 11, p. 113701, 2012.

W. Xie, R. Gomes, T. Aubert, S. Bisschop, Y. Zhu, Z. Hens, E. Brainis, and D. Van Thourhout, "Nanoscale and SingleDot Patterning of Colloidal Quantum Dots," Nano Lett., vol. 15, pp. 7481-7486, 2015.

[79] W. Tittel, H. Zbinden, and N. Gisin, "Quantum cryptography," Rev. Mod. Phys., vol. 74, no. January, pp. 145-195, 2002.

[80] M. Varnava, D. E. Browne, and T. Rudolph, "How Good 
Must Single Photon Sources and Detectors Be for Efficient Linear Optical Quantum Computation?," Phys. Rev. Lett., vol. 60502, no. February, pp. 1-4, 2008.

[81] B. Mahler, P. Spinicelli, S. Buil, X. Quelin, J.-P. Hermier, and B. Dubertret, "Towards Non-Blinking Colloidal Quantum Dots," Nat. Mater., vol. 7, pp. 659-664, 2008.

[82] S. Bisschop, A. Guille, D. Van Thourhout, Z. Hens, and E. Brainis, "Broadband enhancement of single photon emission and polarization dependent coupling in silicon nitride waveguides," Opt. Express, vol. 23, no. 11, pp. 1371313724,2015

Weiqiang Xie received the B.S. degree in applied physics from Xi'an Jiaotong University in 2008, and the M.S. degree in condensed matter physics from Shanghai Jiaotong University in 2011. In 2016, he received the PhD degree in photonics engineering from Ghent University. During his $\mathrm{PhD}, \mathrm{He}$ was working on the development of passive and active silicon/silicon nitride integrated photonics including silicon nanophotonic crystals, silicon nitride platform for low-loss waveguides and on-chip high-quality microresonators, and the integration of silicon nitride photonics with colloidal quantum dots for on-chip lasers.

Yunpeng Zhu received the B.S. degree in Optical information science and technology from Nanjing University of Posts and Telecommunication, Nanjing, China, in 2007, and the M.S. degree in optical engineering from Zhejiang University, Hangzhou, China in 2011. He is currently working towards the Ph.D. degree in Photonics Research Group, Ghent University and IMEC. His research interests include integration of colloidal quantum dots with silicon nitride photonics for on-chip active components.

Suzanne Bisschop Suzanne Bisschop received the B.S. degree in applied physics and the M.S. degree in Photonics from Ghent University, Belgium in 2011 and 2013 respectively. She is currently working toward the Ph.D. degree in the Physics and Chemistry of Nanostructures group at Ghent University. Her research interests include the ultrafast photophysics of colloidal nanocrystals and their integration in silicon nitride photonics for on-chip sources.

Tangi Aubert received a M.S. degree in Chemistry and Physics from Bordeaux University (France) in 2008 and a $\mathrm{PhD}$ degree in Chemistry from Rennes University (France) in 2011. He then was appointed as a post-doctoral researcher at Ghent University (Belgium), focusing on the development of high performing quantum dots and nanoparticles for their application to nano- and biophotonics.

Zeger Hens obtained his $\mathrm{PhD}$ from Ghent University in 2000, which was followed by a post-doctoral stay at the University of Utrecht. In 2002, he returned to Ghent University as a professor and started a research group on colloidal nanocrystals. His work covers the synthesis, processing and characterization of colloidal quantum dots and their application in photonic devices.

Dries Van Thourhout received the degree in physical engineering and the Ph.D. degree fromGhent University, Ghent, Belgium in 1995 and 2000, respectively. From 2000 to 2002, he was with Lucent Technologies, Bell Laboratories, Crawford Hill, working on InP/InGaAsP monolithically integrated devices. In 2002, he joined the Department of Information Technology (INTEC), Ghent University, continuing his work on integrated optoelectronic devices. His research interests include heterogeneous integration by wafer bonding, intrachip optical interconnect and WDM-devices.

Pieter Geiregat received a B.S, M.S. and PhD degree in Engineering Physics from Ghent university in 2008, 2010 and 2015 respectively. During his PhD he was working at the TU Delft (Netherlands) where he first came into contact with ultrafast optical spectroscopy of nanomaterials. In the first year after his $\mathrm{PhD}$ he investigated the application potential of colloidal quantum dots for integrated amplifiers under a H2020 ERC:PoC grant. Currently, he holds a postdoctoral fellowship (FWO Belgium) to investigate the ultrafast photophysics of solution processable nanomaterials and integrated photonic devices made thereof. His research interests include integrated nanophotonics, ultrafast $1 \mathrm{D}$ and $2 \mathrm{D}$ optical spectroscopy of nanoscale materials and strong light-matter coupling. 